3. Kalk E, Goede A, Rose P. Acute arterial thrombosis in acute promyelocytic leukaemia. Clin Lab Haematol. 2003;25(4):267-70.

4. Barbui T, Falanga A. Disseminated intravascular coagulation in acute leukemia. Semin Thromb Hemost. 2001;27(6):593-604.

5. Gupta V, Yi QL, Brandwein J, Lipton JH, Messner HA, Schuh AC et al. Role of all-trans-retinoic acid (ATRA) in the consolidation therapy of acute promyelocytic leukaemia (APL). Leuk Res. 2005; 29(1):113-4.

Avaliação: Editor e dois revisores externos

Conflito de interesse: não declarado

Recebido: 26/05/2006

Aceito após modificações: 23/03/2007

Correspondência: Ricardo Ambrósio Fock

Rua Anchieta, 341

13201-804 - Jundiaí, SP

E-mail:ricfock@hotmail.com

\section{O fator estimulador de colônias granulocitárias (G-CSF) para isquemia cerebral. Uma nova aplicação terapêutica?}

\section{Granulocyte colony-stimulating factor (G-CSF) for ischemic stroke. A new therapeutic modality?}

\author{
Angelo Luiz Maset ${ }^{1}$ \\ Kleber P. Duarte ${ }^{2}$ \\ Oswaldo Tadeu Greco ${ }^{3}$
}

${ }^{1}$ Neurocirurgião - Associação Portuguesa de Beneficência de S.J. Rio Preto-SP; InNeuro - Instituto de Neurocirurgia e Neurociências do Oeste Paulista

${ }^{2}$ Neurocirurgião - Associação Portuguesa de Beneficência de S.J. Rio Preto-SP

${ }^{3}$ Cardiologista. Instituto de Moléstias Cardiovasculares de S.J. Rio Preto-SP

\section{Senhor Editor:}

O G-CSF é uma glicoproteína que foi descrita há mais de vinte anos, possui aprovação do FDA e é comumente utilizada para tratar neutropenia, ${ }^{1}$ ou para transplantes de medula óssea. ${ }^{2,3}$ Incluem-se como fontes de G-CSF os monócitos, células mesoteliais, fibroblastos e células endoteliais. O G-CSF estimula o crescimento de precursores de neutrófilos e regula crucialmente a sobrevivência de neu- trófilos maduros, pós-mitóticos, através da inibição da apoptose..$^{2,3,4}$ Desde o início desta década, vários pesquisadores têm demonstrado a surpreendente atividade do G-CSF no sistema nervoso central (SNC) ${ }^{5-8}$ A administração de GCSF mobiliza células-tronco e progenitoras da medula óssea para o sangue periférico, que por sua vez atravessam a barreira hemato-encefálica (BHE) e se dirigem à área acometida do cérebro. O G-CSF possui claramente um efeito neuroprotetor aparentemente multimodal, incluindo-se a atividade antiapoptóptica em neurônios, ${ }^{5,6}$ regeneração de vascularização, ${ }^{8}$ efeito antiinflamatório ${ }^{9}$ e estimulação de neurogênese endógena. ${ }^{10}$

Estes estudos promissores em animais deram o embasamento necessário para as primeiras utilizações um humanos. Ickenstein ${ }^{11}$ reportou a ausência de efeitos adversos, Shyu e $\mathrm{col}^{12}$ exploraram o potencial terapêutico do G-CSF em A.V.C. agudo isquêmico da artéria cerebral média, utilizandose dos seguintes critérios: diagnóstico de AVC através de ressonância magnética à admissão hospitalar; déficit neurológico avaliado pela escala de Stroke do NIH (NIHSS) e selecionados quando entre 9 e 20; período máximo de inclusão de 7 dias após início do AVC. Num período de 12 meses póstratamento, os pacientes que receberam G-CSF (n=7) apresentaram melhora muito mais expressiva na função neurológica do que os pacientes controle $(n=3)$ em todas as escalas clínicas utilizadas, incluindo NIHSS, escala de Stroke européia, e índice Barthel.

Relatamos neste comunicado nossa experiência com que talvez tenha sido o primeiro paciente brasileiro a fazer uso de G-CSF especificamente para uma patologia intracerebral, a qual se deu logo após a publicação de Shyu. ${ }^{12}$

Apresentamos o relato de caso de paciente de 74 anos de idade com quadro de hemorragia subaracnóidea que evoluiu para isquemia cerebral, com vaso espasmo em território das artérias cerebrais médias e posteriores bilateralmente e sistema vértebro-basilar (isquemia global). A angiografia cerebral dos vasos não mostrou origem do episódio de sangramento. A avaliação neurológica caracterizou um quadro grave de isquemia global escore GCS 3 e NIHSS 25.

Foram realizadas as medidas terapêuticas usuais - manutenção de pressão de perfusão cerebral, medidas antivasoespasmo, controle hidroeletrolítico com hipermagnesemia induzida, e terapia de suporte hemodinâmico e ventilatório gerais. Foi administrado o G-CSF na dose de $15 \mathrm{ug} /$ $\mathrm{kg} / \mathrm{dia} / \mathrm{sc}$ durante cinco dias consecutivos, a partir do $11^{\circ}$ dia da instalação da isquemia global. Foram realizados hemogramas diários e uroanálises freqüentes durante o período de internação na UTI (44 dias). Foram realizadas tomografias computadorizadas seqüenciais (anterior à administração de G-CSF, trinta e sessenta dias após) e repetida angiografia de quatro vasos (oito dias após início de G-CSF). Foi realizado SPECT sessenta dias após início G-CSF.

Observamos as alterações nos exames laboratoriais de rotina, bem como as alterações clínicas e radiológicas num período de follow-up de três meses.

Os leucócitos eram de $11.550 / \mathrm{mm}^{3}$ no dia imediatamente anterior ao G-CSF. Após o uso do G-CSF, os leucócitos se 
elevaram para 30.560 no primeiro dia e atingiram um máximo de 59.250/ $\mathrm{mm}^{3}$ no sexto dia do início da administração (ou um dia após o término da administração de G-CSF) e então foram diminuindo gradativamente até o $10^{\circ}$ dia após o início da administração de G-CSF (10.420 leucócitos/mm³. Não houve nenhuma alteração metabólica durante ou imediatamente após o período de administração de G-CSF.

O paciente apresentou melhora progressiva da escala de avaliação de Glasgow, iniciada no primeiro dia após a administração, com abertura ocular (AO), a qual perdurou. A resposta motora $(\mathrm{RM})$ melhorou ao redor do $50^{\circ}$ dia e a resposta verbal (RV) iniciou-se ao redor de setenta dias. A melhora clínica observada refletiu nas escalas de avaliação. A escala Glasgow inicialmente era de 3, e subiu para 12 na ocasião da alta hospitalar. A escala NIHSS inicialmente era de 25, e caiu para 12 na ocasião da alta hospitalar. Obviamente, a melhora já no primeiro dia não deve ser creditada ao G-CSF, e este comunicado não tem a intenção de discutir a eficácia da droga neste momento, mas sim de salientar a sua segurança.

Em conclusão, o G-CSF se mostrou seguro nas doses aplicadas, e os resultados deste paciente estão em consonância com os resultados citados na literatura. Este relato será publicado eventualmente em revista científica neurocirúrgica.

\begin{abstract}
G-CSF is a FDA-approved drug, commonly utilized in neutropenic patients, long knows for its anti-inflammatory and angiogenic properties and also for its capacity to mobilize stem cells, hematopoiethic and endogen precursors and progenitor cells. Recently neuroprotective effects have been shown in animals and humans and there are publications mentioning its potential therapeutic role in focal ischemia (medial cerebral artery territory). We describe a case of a 74-year-old male patient, victim of a subarachnoid hemorrhage and brain ischemia due to bilateral involvement of medial cerebral arteries and vertebrobasilar vasospasms (overall ischemia), who was submitted to G-C5F therapy. G-CSF did not cause adverse effects, mobilized cells (as was seen by the exponential increase in leukocytes) and possibly contributed to a progressive clinical improvement confirmed using GCS and NIHSS scales. Clinical improvements did not reflect in eithrt CT, MNR or SPECT examinations. This initial experience opens the perspective for G-CSF studies in brain ischemia. Rev. bras. hematol. hemoter. 2007; 29(4):419-420
\end{abstract}

Key words: Ischemic stroke; G-CSF; therapy.

\section{Referências Bibliográficas}

1. Burgess AW, Metcalf D. Characterization of a serum factor stimulating the differentiation of myelomonocytic leukemic cells. Int J Cancer. 1980;26:647-54

2. Welte K, Platzer E, Lu L, Gabrilove JL, Levi E, Mertelsmann R, et al. Purification and biochemical characterization of human pluripotent hematopoietic colony-stimulating factor. Proc Nat Acad Sci USA 1985;82:1526-30.
3. Begley CG et al. Purified colony-stimulating factors enhance the survival of human neutrophils and eosinophils in vitro: a rapid and sensitive microassay for colony-stimulating factors. Blood. 1986, 68:162-166

4. Hu B, Yasui K. Effects of colony stimulating factors (CSFs) on neutrophil apoptosis: possible roles at inflammations site. Int $\mathrm{J}$ Hematolog 66:179-88.

5. Schabitz WR, Kollmar R, Schwaninger $\mathrm{M}$ et al. Europrotective effect of granulocyte colony-stimulating factor after focal cerebral ischemia. Stroke. 2003;34:745-51.

6. Schneider A, Kruger C, Steigleder T et al. The hematopoietic factor G-CSF is a neuronal ligand that counteracts programmed cell death and drives neurogenesis. The Journal of Clinical Investigation. 2005;115(8);2083-98.

7. Six I, Gasan G, Mura E, Bordet R. Beneficial effect of pharmacological mobilization of bone marrow in experimental cerebral ischemia. Eur J Pharmacol 2003;458(3):327-8.

8. Lee ST, Chu K, Jung KH, Ko SY, Kim EH, Sinn DI, et al. Granulocyte colony-stimulating factor enhances angiogenesis after focal cerebral ischemia. Brain Res. 2005;1058(1-2):120-8.

9. Hartung T. Anti-inflammatory effects of granulocyte colonystimulating factor. Curr Opin Hematol. 1998;5(3):221-5.

10. Schneider A, Kuhn HG, Schabitz WR. A role of G-CSF (granulocytecolony stimulating factor) in the central nervous system. Cell Cycle. 2005;4(12):1753-7.

11. Ickenstein G, Haas S, Sauerbruch S et al. Regeneration in acute ischemic stroke (RAIS) with hematopoietic stem cell mobilization (abstract). In: 56 th Annual Meeting of the American Academy of Neurology; 24 April-1 May 2004. San Francisco: American Academy of Neurology;2004. Program No S39.001

12. Shyu WC, Lin SZ, Lee CC et al. Granulocyte colony-stimulating factor for acute ischemic stroke: a randomized controlled trial. CMAJ 2006;174(7):927-33.

Avaliação: Editor e dois revisores externos

Conflito de interesse: não declarado

Recebido: 16/04/2007

Aceito após modificações: 10/05/2007

Correspondência: Ângelo Luiz Maset

Av Pres Juscelino K. Oliveira, 1.001

15091-450 - Jd. Panorama

São José do Rio Preto-SP

E-mail:maset@terra.com.br 